\title{
Pleomorphic Xanthomatous Lesions and CAD in a Family
}

\section{Dwivedi S and Jhamb R*}

Department of Medicine and Preventive Cardiology, University College of Medical Sciences and G.T.B. Hospital, Delhi, India

\section{Case Report}

A 42- year lady presented with precordial chest pain, intermittent claudications and dyspnea of four week duration. She had no past history of diabetes mellitus, hypertension or coronary artery disease (CAD). On examination her blood pressure was 150/100 $\mathrm{mm}$ of $\mathrm{Hg}$ and pulse rate - 84 per minute, regular and had evidence of peripheral vascular disease in bilateral lower limbs. Her body mass index was 26.06 and waist circumference $-86 \mathrm{cms}$. She had extensive bilateral xanthaelasma both in upper and lower eyelids and had distinct arcus (Figure 1). Her cholesterol (399 mg/dl) LDL-cholesterol $(314 \mathrm{mg} / \mathrm{dl})$ and triglycerides $(241 \mathrm{mg} / \mathrm{dl})$ were raised and HDL-cholesterol $(24$ $\mathrm{mg} / \mathrm{dl}$ ) was low. Her electrocardiogram and echocardiography was suggestive of CAD. Doppler study of bilateral lower limbs confirmed evidence of peripheral arterial disease. Family history revealed both parents having suffered CAD and one of his three brothers had past history of cerebrovascular accident. Interestingly both parents and other siblings except one had some form of xanthoma. One of the elder brothers who had suffered stroke was detected to have both xanthaelasma palpebrum as well as extensive xanthomas spread over back of shoulders and gluteal region (Figure 2). He too had alarmingly raised serum cholesterol (542mg/dl) and a low HDL ( $24 \mathrm{mg} / \mathrm{dl})$.

Both cases are classical examples of familial mixed dyslipidaemia with manifest cutaneous markers of CAD [1]. What makes these twin cases interesting is the pleomorphic presentation of xanthomatous

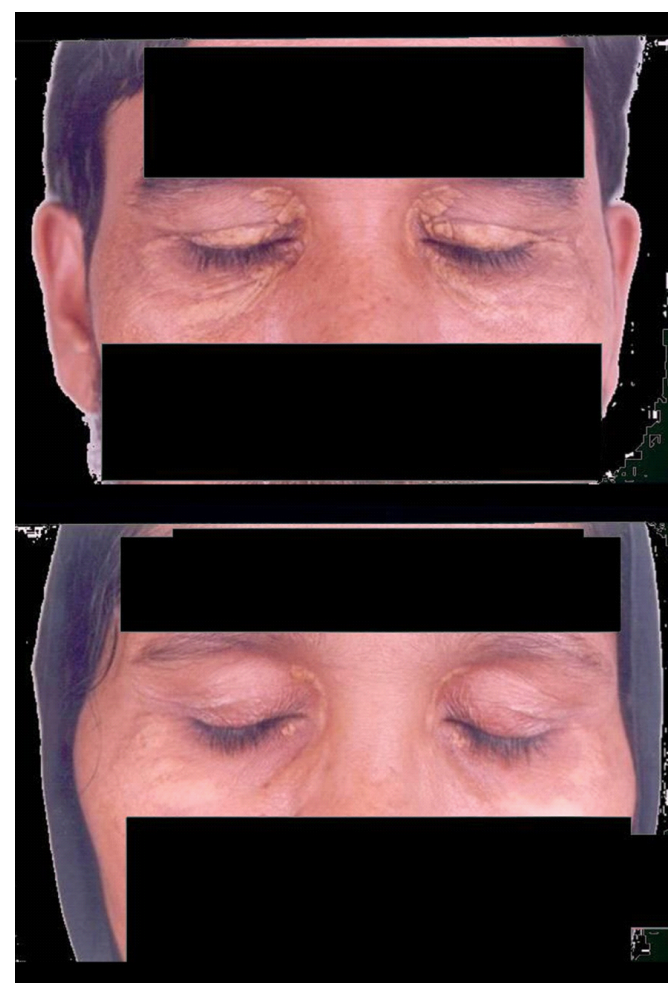

Figure 1: Index case and her brother showing bilateral xanthaelasma palpebrum in both eyelids.
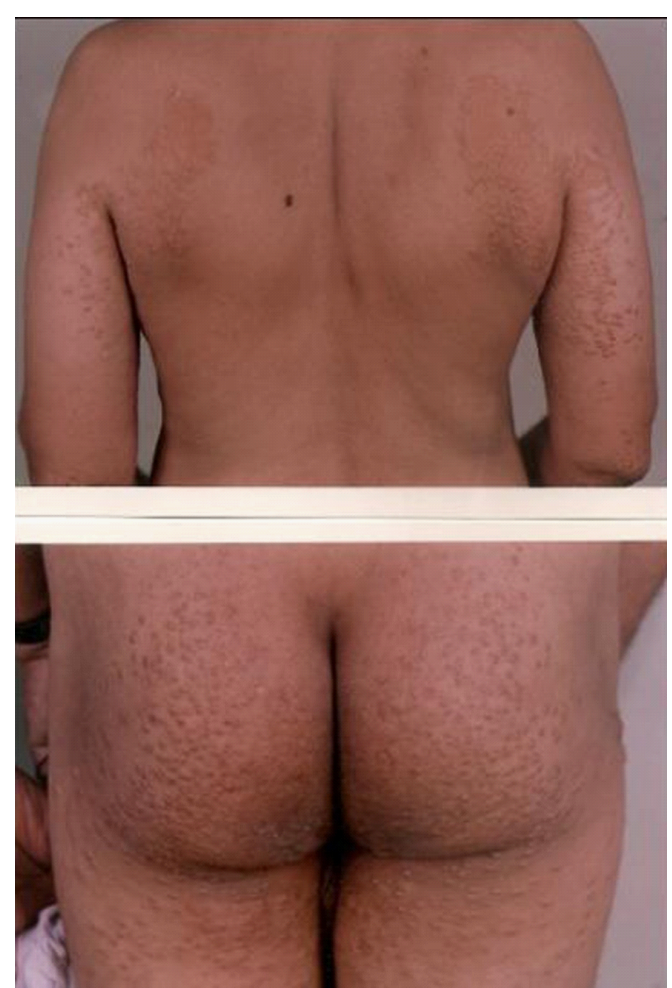

Figure 2: Xanthomas spread over back and gluteal region in brother of the index case.

lesions in two members of same family. In index case it was xanthaelasma palpebrum and arcus juvenalis while the elder brother had both xanthaelasma palpebrum and extensive xanthomas.

Xanthomas usually are associated with disturbance of lipid metabolism [2]. Mechanism of accumulation of lipids in skin morphs is similar to the development of atheroma. Such lesions can have many types including xanthaelasma palpebrum, tuberous xanthomas, tendinous xanthomas, eruptive xanthomas, plane xanthomas, diffuse plane xanthomatosis, xanthoma disseminatum and giant gluteal xanthomas. Gluteal xanthomas are a matter of clinical curiosity because of their peculiar location in the gluteal region and are likely to be missed completely if not looked for diligently [3]. In both patients cutaneous

*Corresponding author: Rajat Jhamb, Department of Medicine and Preventive Cardiology, University College of Medical Sciences and G.T. B. Hospital, Delhi, India, E-mail: rajatjhamb@yahoo.com

Received December 01, 2011; Accepted January 26, 2012; Published January 31, 2012

Citation: Dwivedi S, Jhamb R (2012) Pleomorphic Xanthomatous Lesions and CAD in a Family. J Blood Disord Transfus 3:115. doi:10.4172/2155-9864.1000115

Copyright: @ 2012 Dwivedi S, et al. This is an open-access article distributed under the terms of the Creative Commons Attribution License, which permits unrestricted use, distribution, and reproduction in any medium, provided the original author and source are credited. 
Citation: Dwivedi S, Jhamb R (2012) Pleomorphic Xanthomatous Lesions and CAD in a Family. J Blood Disord Transfus 3:115. doi:10.4172/21559864.1000115

Page 2 of 2

lesions predated the atherosclerotic vascular disease. Obviously, if a patient has any evidence of xanthoma in his/her body; further search should be done to locate xanthomas in other locations. Besides other first degree relatives should be examined for evidence of xanthomatous lesions and associated cardiovascular disorders. They could well be a valuable marker of underlying atherosclerotic vascular disease.

\section{References}

1. Dwivedi S, Aggarwal A (2010) The skin in general medicine. Clin Med 10: 306.

2. Goldsmith LA (2003) Xanthomas and lipoprotein disorders. Fitzpatrick's dermatology in general medicine. McGraw Hill, New York, USA

3. Dwivedi S, Aggarwal A, Sharma V, Dwivedi G (2010) Giant gluteal xanthomas. Circulation 121: 1866-1867. 